\title{
Nucleotide Sequence of the $n$ fo Gene of Escherichia coli K-12
}

\author{
SUSAN M. SAPORITO AND RICHARD P. CUNNINGHAM* \\ Department of Biological Sciences, State University of New York at Albany, Albany, New York 12222
}

Received 31 May 1988/Accepted 10 August 1988

\begin{abstract}
The nfo gene of Escherichia coli K-12 which encodes endonuclease IV has been sequenced. The predicted gene product has a molecular weight of 31,562 , in good agreement with the size of the gene product estimated by maxicell analysis. The nfo promoter was mapped by primer extension of in vivo transcripts. Inspection of the nucleotide sequence revealed no regions of potential secondary structure corresponding to a transcriptional terminator downstream from the structural gene; however, there was a potential open reading frame immediately downstream from the nfo structural gene.
\end{abstract}

Escherichia coli contains two AP endonucleases that cleave 5' to AP sites, exonuclease III (22) and endonuclease IV (15). Other lesions recognized by these enzymes are a group of $3^{\prime}$ blocked termini including 3 '-phosphates and $3^{\prime}$-phosphoglycolate esters $(7,10)$. Urea- $N$-glycosides are endonucleolytically attacked by exonuclease III (11), whereas endonuclease IV preferentially attacks modified AP sites created by bleomycin and neocarzinostatin $(19,20)$. Exonuclease III is the major activity in uninduced cells (16), whereas endonuclease IV can be induced by agents that generate superoxide radical ions (3). Experiments with mutants deficient in either or both of these enzymes suggest that endonuclease IV plays a relatively minor role in the repair of alkylation damage and the repair of AP sites but plays a major role in the recovery of cells treated with bleomycin and $t$-butyl hydroperoxide (5). These differences may be explained by nonoverlapping enzymatic activities or by differences in the genetic structure and regulation of the genes coding for these enzymes. We have sequenced the $x$ th gene (26) and the nfo gene to understand the structure and function of the genes and gene products.

\section{MATERIALS AND METHODS}

Bacterial strains and growth conditions. All experiments were performed with $E$. coli RPC51 except for maxicell analysis, which was performed with strain DR1984 (24). RPC51 has the following genotype: endA thi hsdR $\Delta(s r l R-$ recA)306. Subcloning was done in the plasmid pBR322. A derivative of $\mathrm{pBR} 325$ was used for maxicell analysis. Plasmid pUC4K (30) was the source of a restriction fragment containing a gene specifying kanamycin resistance. Media and growth of cells were as previously described (4).

Enzymes, radioisotopes, and synthetic oligonucleotides. $\mathrm{Re}$ striction endonucleases, exonuclease BAL 31, T4 DNA ligase, T4 polynucleotide kinase, and RNase-free DNase I were purchased from Boehringer Mannheim Biochemicals or International Biotechnologies Inc. DNA polymerase I Klenow fragment, avian myeloblastosis virus reverse transcriptase, and the Sequenase sequencing kit were purchased from United States Biochemical Corp. RNase inhibitor was purchased from Promega Biotec Co. $\left[\alpha-{ }^{32}\right.$ P]dATP, $[\alpha-$ $\left.{ }^{32} \mathrm{P}\right] \mathrm{dCTP},\left[\gamma^{32} \mathrm{P}\right] \mathrm{ATP}$, and $\mathrm{L}-\left[{ }^{35} \mathrm{~S}\right]-$ methionine were purchased from Dupont, NEN Research Products. Synthetic oligonucleotide linkers were purchased from Pharmacia Fine Chemicals. The M13 universal sequencing primers were purchased from International Biotechnologies.

\footnotetext{
* Corresponding author.
}

DNA substrate and AP endonuclease assay. ${ }^{3} \mathrm{H}$-labeled partially depyrimidinated T4 phage DNA (6) and sonicates of log-phase cells were prepared and diluted as previously described (5). The acid solubility assay for endonuclease IV was performed as described by Cunningham et al. (5).

Analysis of plasmid-specific proteins. Maxicell analysis of plasmid-coded proteins was performed by the procedure of Sancar et al. (23) as described by Rodriguez and Tait (21).

Nucleotide sequencing. Restriction fragments of plasmid pRPC124 and its deletion derivatives were subcloned into M13 sequencing vectors (18) mp18, mp19, um20, and um21. Single-stranded DNA template DNA was prepared according to the International Biotechnologies sequencing manual. Nucleotide sequence was determined by the dideoxy-chain termination method $(25,28)$. Nucleotide sequences were compiled and analyzed with the gel reader and the IBI/ Pustell DNA and protein sequence analysis system from International Biotechnologies.

Isolation and manipulation of DNA. Plasmids were amplified with chloramphenicol or spectinomycin and purified by a modified alkaline lysis procedure (1). The conditions for restriction endonuclease and BAL 31 exonuclease digestion were those suggested by the manufacturers. DNA linkers were added as previously described (27). Partial digestion of plasmids was accomplished by removing samples from a standard reaction at various times and stopping the reaction with excess trisodium EDTA. The products from partial or complete restriction digestions were separated on agarose gels, and the appropriate fragments were excised and purified with the Geneclean kit (Bio 101, Inc.). The ligation of linear plasmid molecules after the addition of linkers, ligation of DNA fragments into a cleaved vector (17), and DNA transformation into calcium-treated cells (21) were previously described.

Isolation of RNA and primer extension. The isolation of cellular RNA and primer extension of cellular RNA has been previously described (26).

Other methods. Protein concentrations were determined by the method of Bradford (2).

\section{RESULTS}

Subcloning of the nfo gene. We have previously identified a clone bearing a ColE1 hybrid plasmid from the ClarkeCarbon collection, pLC38-27, which overproduces endonuclease IV. The nfo gene was subcloned and localized to a 3.9-kilobase BamHI-HindIII fragment on the plasmid pWB21 (5). The approximate position of the gene within this fragment was determined by creating insertions of the kana- 


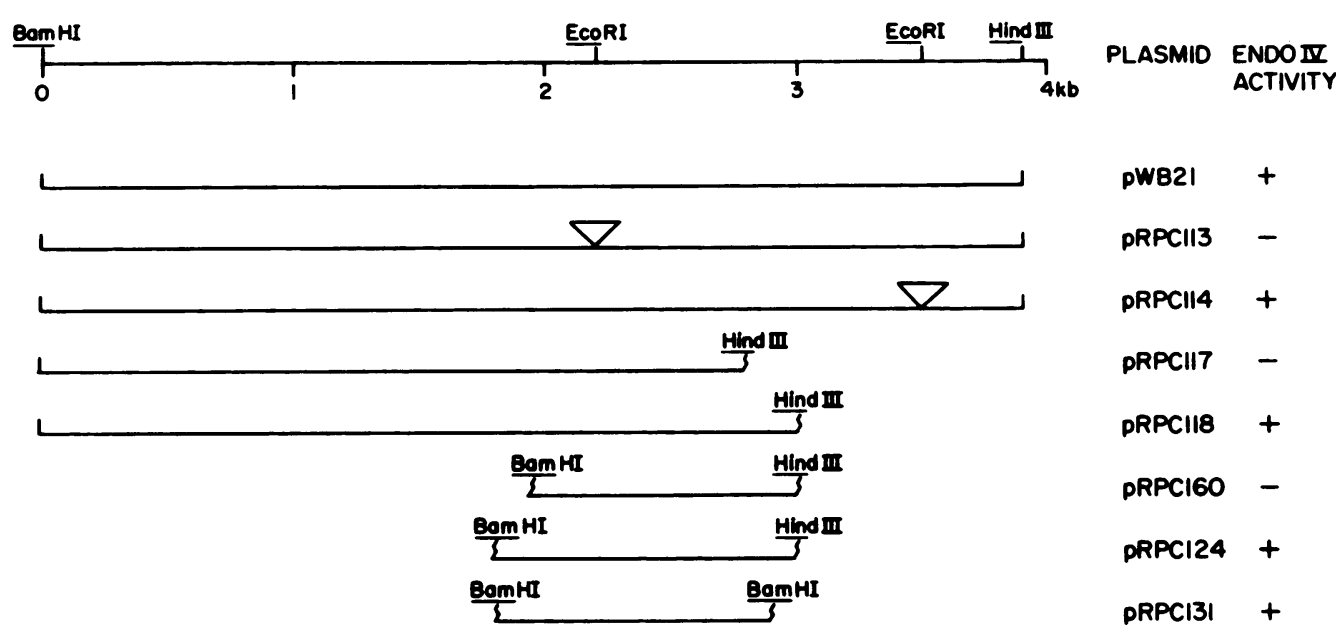

FIG. 1. Physical map of the plasmid pWB21 and the subclones used to localize the nfo gene. The map is of the insert which replaces the BamHI-HindIII fragment of pBR322. Vertical lines represent restriction sites used for subcloning into pBR322. A triangle represents an inserted kanamycin resistance gene. Jagged vertical lines with new restriction sites indicate that Bam HI or HindIII linkers were added to the ends of fragments created by digestion with nuclease BAL 31. The plasmids and their properties are tabulated on the right. The ability of plasmids to express endonuclease IV was determined by enzyme assay. kb, kilobases.

mycin resistance gene of pUC4K in the EcoRI sites of pLC38-27 (5) or pWB1 (Fig. 1). These insertion mutations placed the nfo gene in the center of the 3.9-kilobase BamHIHindIII fragment. To isolate the nfo gene on a smaller piece of DNA, we created BAL 31 deletions that varied by 200 base pairs (bp) from the HindIII site of pWB21. HindIII linkers were added to the deletion plasmids, and the religated plasmids were transformed into RPC51. Strains carrying pRPC118, but not those carrying pRPC117, overproduced endonuclease IV (Fig. 1). We then created a series of BAL 31 deletions varied by $200 \mathrm{bp}$ from the BamHI site of pRPC118. Plasmid pRPC124 but not plasmid pRPC160 was able to overproduce endonuclease IV in RPC51. Plasmid pRPC124 is 5,200 bp in length. To create a BamHI fragment carrying the nfo gene, we created a 50-bp BAL 31 deletion from the HindIII site of pRPC124 and added BamHI linkers. Plasmid pRPC131 contains the nfo gene on a 1.15-kilobase BamHI fragment (Fig. 1).

Characterizing the nfo gene product. The $n f o$ gene that we have subcloned causes the overproduction of endonuclease IV. The nfo gene may be the structural gene for endonuclease IV, or it may be some other gene required for the expression of the endonuclease IV gene. Endonuclease IV has an estimated molecular size of approximately 33 kilodaltons (kDa) based upon its sedimentation coefficient and its Stokes radius (15) and of $31 \mathrm{kDa}$ based upon denaturing polyacrylamide gel electrophoresis (13). We determined the size of the gene product produced by the cloned $n f o$ gene by using maxicell analysis. The pBR322-nfo derivative we constructed, pRPC124, expressed a $\beta$-lactamase that is approximately $30 \mathrm{kD}$. To eliminate this gene product, which could obscure endonuclease IV on gels, we created a new vector. We started with pBR325 and made a small deletion in the amp gene by cutting with PstI and digesting with BAL 31, followed by blunt end ligation. The resulting plasmid, pRPC125, did not express $\beta$-lactamase (Fig. 2, lane 4) as compared with pBR322 and pBR325 (lanes 2 and 3). Plasmid pRPC125 did express a new protein of approximately $26 \mathrm{kDa}$ which may be a shortened $\beta$-lactamase. The BamHI fragment from pRPC131 was cloned into the unique BamHI site of pRPC125 in both orientations to produce plasmids pRPC134 and pRPC135. Both plasmids produced a polypep- tide of $29 \mathrm{kD}$ (Fig. 2, lanes 5 and 6). These results suggest that we have cloned the structural gene for endonuclease IV and its promoter.

Nucleotide sequence of the nfo gene. The sequence of the 1,200-bp BamHI-HindIII fragment in pRPC124 was determined for both strands from overlapping DNA fragments. A detailed restriction map and the specific fragments sequenced are shown in Fig. 3. A DNA sequence of 1,020 nucleotides including the nfo gene is shown in Fig. 4. An open reading frame (ORF) starting at nucleotide 82 with an ATG codon and ending at nucleotide 937 with a TAA stop codon was identified. The identified ORF had a codon usage consistent with the nonrandom codon usage found for a number of $E$. coli genes. Upstream of this ORF was a potential -10 hexamer at nucleotides 38 through 43 and a potential -35 hexamer at nucleotides 14 through 19 with a spacing of 17 nucleotides, which is consistent with an $E$. coli

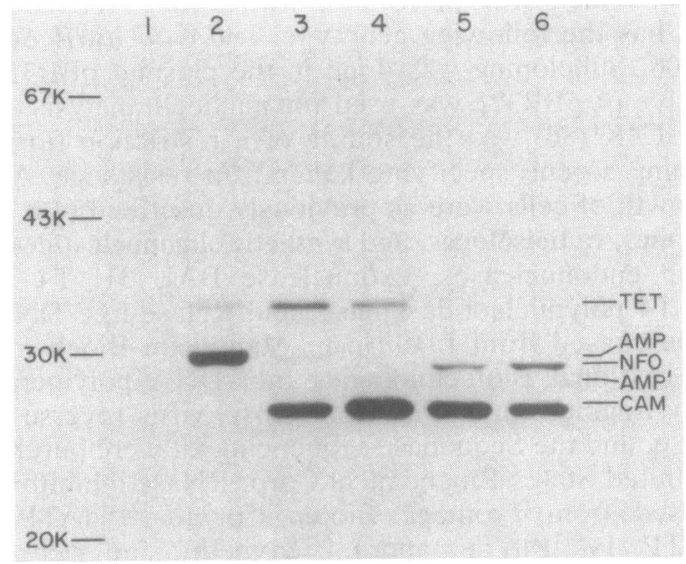

FIG. 2. Sodium dodecyl sulfate-polyacrylamide gel electrophoresis of proteins produced by plasmids bearing the nfo gene in strain DR1984. Proteins were labeled with $\left[{ }^{35} S\right]$ methionine by the maxicell technique. Lanes: 1, no plasmid; 2, pBR322; 3, pBR325; 4, pRPC125; 5, pRPC134; 6, pRPC135. TET, Tetracycline resistance gene product; AMP, $\beta$-lactamase, NFO, nfo gene product; AMP' $^{\prime}$ shortened $\beta$-lactamase; CAM, chloramphenicol transacetylase. 


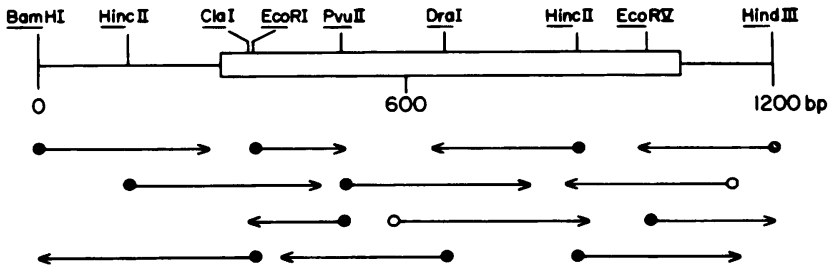

FIG. 3. Strategy and restriction sites used for sequencing the $n f o$ gene. The restriction fragment shown is a 1,200 -bp fragment from pRPC124. Arrows indicate the direction and extent of each sequence determination: $\bullet$, start sites corresponding to internal restriction sites; $O$, start sites created by digestion with nuclease BAL 31. The nfo structural gene is indicated ( $\square$ ) at the top. The entire sequence was derived from overlapping clones sequenced in both directions.

promoter recognized by the $\sigma^{70}$ subunit of RNA polymerase (9). A potential ribosome-binding site of AGGAG was found at nucleotides 70 through 74 , seven bases away from the initiation codon. There was no region of dyad symmetry $3^{\prime}$ to the stop codon, which would be expected of a transcriptional terminator. There was a start codon at nucleotides 942 through 944 followed by an ORF that extends to the end of the sequenced fragment. This ORF also had a codon usage consistent with the nonrandom codon usage found in $E$. coli genes.

The ORF from nucleotides 82 to 937 would code for a polypeptide of 285 amino acids with a calculated molecular mass of $31,562 \mathrm{Da}$, which is consistent with the values derived from analysis of the purified protein and also consistent with the value we determined from maxicell analysis. The N-terminal amino acid sequence of endonuclease IV has been determined for the first six amino acids (13) and is in perfect agreement with our predicted sequence. The amino acid composition of endonuclease IV has been determined (B. Demple, personal communication) and agrees with our predicted amino acid composition. These results establish the identified ORF as the structural gene for endonuclease IV.

Mapping the nfo promoter. Analysis of the DNA sequence $5^{\prime}$ of the nfo gene revealed a potential promoter. To identify the in vivo site of transcriptional initiation, we mapped the nfo promoter by primer extension mapping of nfo transcripts. Cellular RNA from a strain bearing pRPC124 to enrich for $n f o$ transcripts was used as a source of mRNA. A synthetic primer 17 nucleotides long complementary to nucleotides 122 through 138 was $5^{\prime}{ }^{32} \mathrm{P}$ labeled, annealed to cellular RNA, and extended with reverse transcriptase. This primer was also annealed to an M13 clone carrying a portion of the antisense strand of the nfo gene, and a sequencing ladder was prepared by the dideoxy method. The DNA fragments extended by reverse transcriptase were displayed on a sequencing gel (Fig. 5). The fragment generated from reverse transcription of the primer annealed to RNA (lane 5) ended at C48 (lane 4) with minor sites at G46 and G47 (lane 1) and A49 (lane 3). Since this is the sequence of the sense strand, transcription initiates at G48 with minor start sites at C46, C47, and T49. This start site is consistent with the location of the predicted promoter.

\section{DISCUSSION}

We sequenced the gene coding for endonuclease IV of $E$. coli. An ORF coding for a predicted polypeptide of 31,562 $\mathrm{Da}$ was found. The molecular mass of endonuclease IV was

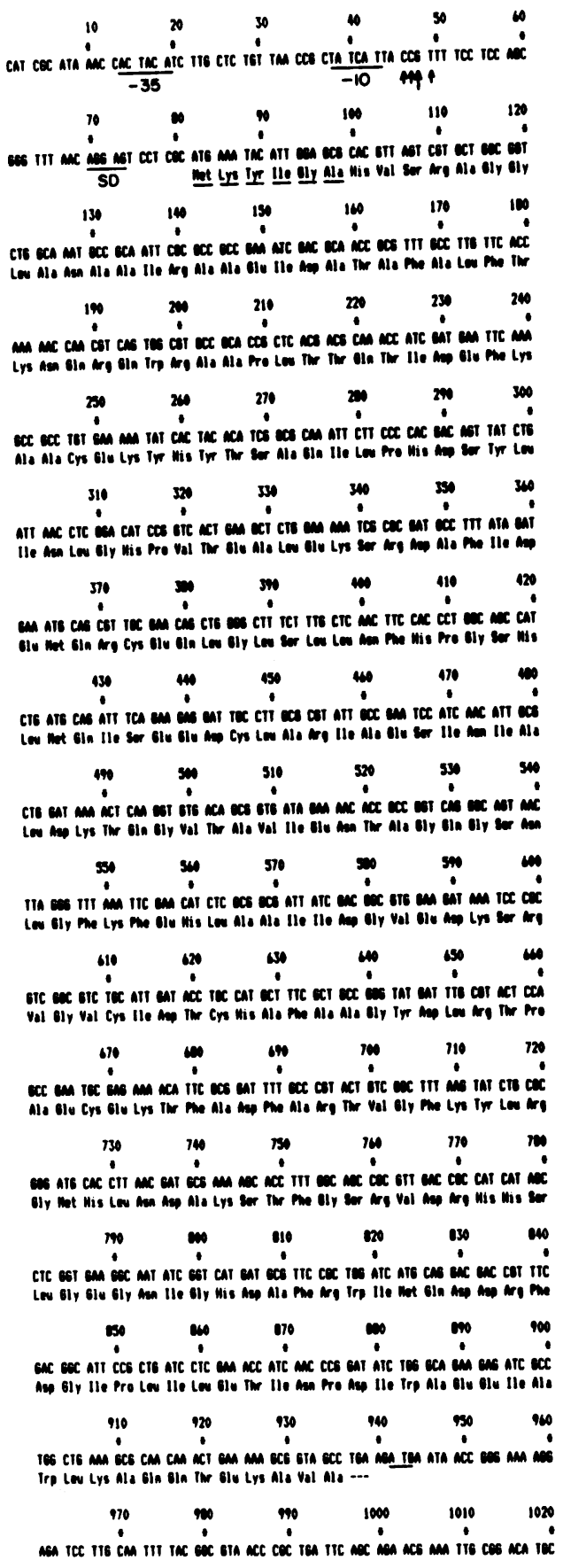

FIG. 4. Nucleotide sequence of the nfo gene and deduced amino acid sequence of endonuclease IV. The DNA sequence of the antisense strand is shown; numbering is from the $5^{\prime}$ end. The proposed -10 and -35 hexamers of the nfo promoter and the Shine-Dalgarno site are underlined and labeled. The arrows below nucleotides 46,47 , and 49 indicate minor transcriptional initiation start sites, and the larger arrow under nucleotide 48 indicates the major initiation site. The potential start codon of the downstream ORF at nucleotides 942 through 944 is underlined. The underlined amino acid residues are those confirmed by protein sequence analysis of endonuclease IV (13). 


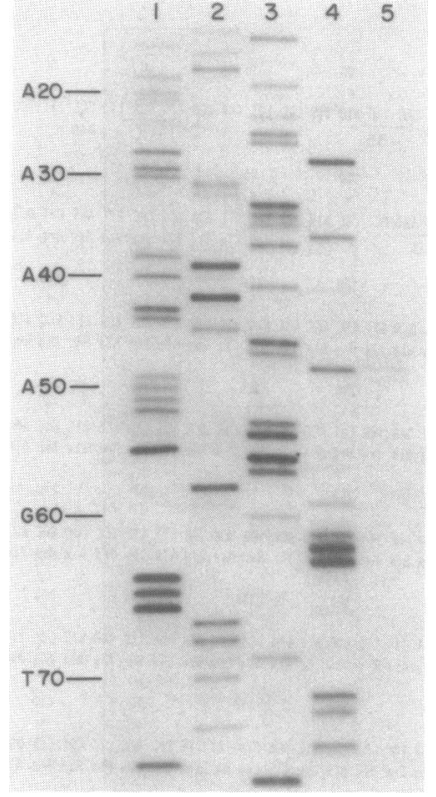

FIG. 5. Primer extension mapping of the nfo promoter. The autoradiogram of an $8 \%$ polyacrylamide gel used to analyze a DNA primer extended by reverse transcriptase is shown. Equivalent amounts of ${ }^{32} \mathrm{P}$-end-labeled DNA primer were used for both the primer extension and the sequencing ladder. The sequencing ladder of the reverse strand was generated by dideoxy sequencing of $\mathrm{A}$ (lane 1), T (lane 2), G (lane 3), and C (lane 4) of the Bam HI-ClaI restriction fragment from $\mathrm{pRPC} 124$ by reverse transcriptase. For the primer extension reaction $20 \mu \mathrm{g}$ of total RNA isolated from RPC51 carrying pRPC124 was used (lane 5). The numbering of the nucleotide positions is in agreement with the complementary sequence in Fig. 4.

determined to be 31 to $33 \mathrm{kDa}$, and we determined that the molecular mass of the $n f o$ gene product is $29 \mathrm{kDa}$. We verified that this ORF is the structural gene for endonuclease IV by comparing the predicted $\mathrm{N}$-terminal amino acid sequence with that determined for purified protein and by comparing the predicted amino acid composition with that determined for purified protein. There is excellent agreement of the predicted sequence and composition with the experimentally determined sequence and composition.

We identified a potential promoter for the nfo gene and found by using primer extension analysis that it serves as the promoter in vivo. This promoter has a -10 hexamer with homology to the consensus sequence. The -35 site of ACTACA does not have significant homology with the consensus -35 site of TGGACA, especially in the highly conserved first three bases. The nfo gene is induced by superoxide radical ions. The inducibility of the gene and the lack of consensus at the -35 site suggest that a positive regulatory protein might be involved in gene regulation. Manganese-superoxide dismutase is also induced by superoxide radical ions (8), and the manganese-superoxide dismutase gene has been sequenced (29). We examined the sequences upstream of the sodA gene and compared them with sequences upstream of the nfo gene to determine whether there is a potential consensus sequence for the binding of a regulatory protein. We found no sequences of significant homology in the two upstream regions.

We found no regions of dyad symmetry indicative of a terminator downstream of the nfo gene. There was an ORF starting at nucleotide 942 with an ATG and extending for 144 nucleotides. The codon usage for the first 48 codons of the ORF is consistent with that found for $E$. coli genes. We created $\beta$-galactosidase fusions to this ORF and found that $\beta$-galactosidase activity was induced by methyl viologen (unpublished observations). Our data strongly suggest that the nfo gene is the proximal gene in an operon. Experiments to clone and characterize the operon are in progress.

The average hydropathy for endonuclease IV is -0.23 , and it contains $23.5 \%$ polar amino acids. A hydropathy profile for endonuclease IV by the method of Kyte and Doolittle (12) was typical of a soluble protein. We were unable to find any degree of similarity between exonuclease III and endonuclease IV at the primary level of protein structure. In addition, a computer search with the search algorithm of Lipman and Pearson (14) of the National Biochemical Research Foundation protein sequence data library did not reveal any proteins with extensive similarity to endonuclease IV.

The roles that exonuclease III and endonuclease IV play in DNA repair are overlapping in some respects yet may also be quite different depending upon the DNA-damaging treatment. Although the two enzymes are similar in function, they are different in structure and have nonoverlapping activities. Endonuclease IV appears to be the first gene in an operon, whereas exonuclease III appears to be a single gene. Endonuclease IV is induced by agents which generate superoxide radical anions, whereas exonuclease III has not been shown to be subject to regulation by DNA-damaging treatments. Our knowledge of the role that these enzymes play in DNA repair requires further studies on their enzymatic functions and also on the structure and regulation of the $x$ th gene and the nfo operon.

\section{ACKNOWLEDGMENTS}

This work was supported by Public Health Service grant GM33346 (to R.P.C.) from the National Institute of General Medical Sciences.

We thank Nadrian Seeman of the State University of New York at Albany for the synthesis of a synthetic oligonucleotide, Bruce Demple of Harvard University for providing us with the N-terminal sequence and the amino acid composition of purified endonuclease IV, and Linda Welch for preparation of the manuscript.

\section{LITERATURE CITED}

1. Birnboim, H. C., and J. Doly. 1979. A rapid alkaline extraction procedure for screening recombinant plasmid DNA. Nucleic Acids Res. 7:1513-1523.

2. Bradford, M. M. 1976. A rapid and sensitive method for the quantitation of microgram quantities of protein utilizing the principle of protein-dye binding. Anal. Biochem. 72:248-254.

3. Chan, E., and B. Weiss. 1987. Endonuclease IV of Escherichia coli is induced by paraquat. Proc. Natl. Acad. Sci. USA 84: 3189-3913.

4. Clyman, J. C., and R. P. Cunningham. 1987. Escherichia coli $\mathrm{K}-12$ mutants in which viability is dependent on recA function. J. Bacteriol. 169:4203-4210.

5. Cunningham, R. P., S. M. Saporito, S. G. Spitzer, and B. Weiss. 1986. Endonuclease IV (nfo) mutant of Escherichia coli. J. Bacteriol. 168:1120-1127.

6. Cunningham, R. P., and B. Weiss. 1985. Endonuclease III (nth) mutants of Escherichia coli. Proc. Natl. Acad. Sci. USA 82: $474-478$.

7. Demple, B., A. Johnson, and D. Fung. 1986. Exonuclease III and endonuclease IV remove 3' blocks from DNA synthesis primers in $\mathrm{H}_{2} \mathrm{O}_{2}$-damaged Escherichia coli. Proc. Natl. Acad. Sci. USA 83:7731-7735.

8. Hassan, H. M., and I. Fridovich. 1979. Regulation of the synthesis of superoxide dismutase in Escherichia coli. Induction by methyl viologen. J. Biol. Chem. 252:7667-7672. 
9. Hawley, D., and W. McClure. 1983. Compilation and analysis of Escherichia coli promoter DNA sequences. Nucleic Acids Res. 11:2237-2256.

10. Henner, W. D., S. M. Grunberg, and W. A. Haseltine. 1983. Enzyme action at 3' termini of ionizing radiation-induced DNA strand breaks. J. Biol. Chem. 258:15198-15205.

11. Kow, Y. W., and S. S. Wallace. 1985. Exonuclease III recognizes urea residues in oxidized DNA. Proc. Natl. Acad. Sci. USA 82:8354-8358.

12. Kyte, J., and R. F. Doolittle. 1982. A simple method for displaying the hydropathic character of a protein. J. Mol. Biol. 157:105-132.

13. Levin, J. D., A. W. Johnson, and B. Demple. 1988. Homogeneous Escherichia coli endonuclease IV. Characterization of an enzyme that recognizes oxidative damage in DNA. J. Biol. Chem. 263:8066-8071.

14. Lipman, D. J., and W. R. Pearson. 1985. Rapid and sensitive protein similarity searches. Science 237:1435-1441.

15. Ljungquist, S. 1977. A new endonuclease from Escherichia coli acting at apurinic sites in DNA. J. Biol. Chem. 252:2808-2814.

16. Ljungquist, S., T. Lindahl, and P. Howard-Flanders. 1976. Methyl methane sulfonate-sensitive mutant of Escherichia coli deficient in an endonuclease specific for apurinic sites in deoxyribonucleic acid. J. Bacteriol. 126:646-653.

17. Maniatis, T., E. F. Fritsch, and J. Sambrook. 1982. Molecular cloning: a laboratory manual. Cold Spring Harbor Laboratory, Cold Spring Harbor, N.Y.

18. Norrander, J., T. Kempe, and J. Messing. 1983. Construction of improved M13 vectors using oligonucleotide directed mutagenesis. Gene 26:101-106.

19. Povirk, L. F., and I. H. Goldberg. 1985. Endonuclease-resistant apyrimidinic sites formed by neocarzinostatin at cytosine residues in DNA: evidence for a possible role in mutagenesis. Proc. Natl. Acad. Sci. USA 82:3182-3186.
20. Povirk, L. F., and C. W. Houlgrave. 1988. Effect of apurinic/ apyrimidinic endonucleases and polyamines on DNA treated with bleomycin and neocarzinostatin: specific formation and cleavage of closely opposed lesions in complementary strands. Biochemistry 27:3850-3857.

21. Rodriguez, R. L., and R. Tait. 1983. Recombinant DNA techniques. Addison-Wesley Co., New York.

22. Rogers, S. G., and B. Weiss. 1980. Exonuclease III of Escherichia coli $\mathrm{K}-12$, an AP endonuclease. Methods Enzymol. 65: 201-211.

23. Sancar, A., A. M. Hack, and W. D. Rupp. 1979. Simple method for identification of plasmid-coded proteins. J. Bacteriol. 138: 692-693.

24. Sancar, A., and W. D. Rupp. 1979. Cloning of $u v r A$, lexC and $s s b$ genes of Escherichia coli. Biochem. Biophys. Res. Commun. 90:123-129.

25. Sanger, F., S. Nicklen, and A. R. Coulson. 1977. DNA sequencing with chain-terminating inhibitors. Proc. Natl. Acad. Sci. USA 74:5463-5467.

26. Saporito, S. M., B. J. Smith-White, and R. P. Cunningham. 1988. Nucleotide sequence of the $x$ th gene of Escherichia coli K-12. J. Bacteriol. 170:4542-4547.

27. Seth, A. 1984. A new method for linker ligation. Gene Anal. Tech. 1:99-103.

28. Tabor, S., and C. C. Richardson. 1987. DNA sequence analysis with a modified bacteriophage T7 RNA polymerase. Proc. Natl. Acad. Sci. USA 84:4767-4771.

29. Takeda, Y., and H. Avila. 1986. Structure and gene expression of the E. coli Mn-superoxide dismutase gene. Nucleic Acids Res. 14:4577-4589.

30. Viera, J., and J. Messing. 1982. The pUC plasmids, an M13mp7derived system for insertion mutagenesis and sequencing with synthetic universal primers. Gene 19:259-268. 